\title{
Contemporary challenges and comprehensive modifications in existing mobile IPv6
}

\author{
Purnendu Shekhar Pandey ${ }^{1 *}$ K.S Verma ${ }^{2}$ \\ $1^{1 *}$ Department of ICT, Gautam Buddha University, Gr.Noida, Gautam Buddha Nagar (UP), INDIA. \\ ${ }^{2}$ Department of Electrical Engineering, , K.N.I.T Sultanpur (UP), INDIA. \\ E-mails: shekhar.rockwell@gmail.com (Purnendu ${ }^{1},{ }^{1}$ Corresponding author), ksv211@rediffmail.com (K.S.Verma)
}

\begin{abstract}
Several fast handover and better resource utilization techniques were proposed such as L3- Driven Fast Handover and Resource Efficient Care-of-Address Provisioning earlier but these techniques were found beset with various limitations in the view of their fulfilling the fast handover requirement and the demand for fast and real dynamic world applications. To cope up with this demand this paper not only discusses the problem areas within these two techniques but also proposes some viable solutions to the problems MIPv6 is riddled with at present so that they can enhance their capabilities in terms of latency and better utilization of their resources.
\end{abstract}

Keywords: Handover, CoA, Home Agent, Foreign Agent, MIPv4, MIPv6

DOI: http://dx.doi.org/10.4314/ijest.v4i1.15S

\section{Introduction}

Widespread use of wireless devices and the need for uninterrupted communication while moving from one subnet to another guided necessity for continuous mobility of internet. It is world-wide practice universally applied so that the user enjoys continuous internet connectivity and avoids rebooting their application. Internet connectivity is based on certain protocols such as Transmission Control Protocol (TCP) and Internet Protocol (IP).Such protocols require that each Mobile Node (MN) should have a unique IP Address for identifying the physical location of the MN for setting up the connection but mobility of the MN is only achieved if its IP address keeps on changing from one subnet to another subnet. To overcome this problem Mobile IP was developed, which removes the problem by providing Mobile Node with two types of addresses, i.e. a Home Address (HA) that does not change as the node moves and a Care-of-Address (CoA) that keeps on changing as the MN moves from one subnet to other (P. Calhoun et al. ,2002). Mobility support was first incorporated using Internet Protocol Version 4 (IPv4) bringing forth MIPv4,later on Internet Engineering Task Force (IETF) developed another Mobile IP that is MIPv6 (Bi-Lynn et al, 2007) .The major factor that led to switching from MIPv4 to MIPv6 are as follows:

- "Route Optimization" in MIPv6

- "Triangular Routing” problem existed in MIPv4.

But, MIPv6 per se has also got some bottlenecks .The major challenge behind MIPv6 is to reduce the handover latency (It is the time Mobile Node takes while switching from one subnet to another.).To reduce this latency problem various ways are followed which would be dealt with in section 3 of the paper and also provide, accordingly, some viable up-gradation would be mooted on the existing solution in section 4 of the paper. 


\section{MIPv6 Basic Operations: A Quick Look}

MIPv6 protocol was developed which allows MN to be communicated and reachable while moving around in IPv6 internet (D.Johnson et al, 2011). MIPv6 performs mobility with the help of three added addresses-

- Home Address(static address)

- Care-of-Address(CoA)

- Link Layer Address(Prefix)

Packets are to be transmitted to the Mobile Node using HA without actually bothering about Mobile Node current point of attachment to the internet. Only HA is not sufficient at all. The CoA generated by the Foreign Agent (A router on the network that the Mobile Node is visiting) is also required. So, after having known this address a correspondent node or Generic or Home Agent (A router on a Mobile Node's home network which maintains current location information for the mobile) can send the desired packets to the MN. So, these two addresses are sufficient enough for the proper flow of the packets. Now, the question arises as to what for the Link Layer address is used. In brief, it is used for the better handover (handover or handoff refers to the process of transferring an ongoing call or data session from one channel connected to the another) (Christian Vogt et al, 2009).It is the process that would be duly followed and discussed in course of the paper.

When a Mobile Node moves from Home Agent to Foreign Agent as shown in Fig.1 the following process happens before the actual communication starts between Mobile Node and correspondent node (A peer node with which a Mobile Node is communicating. It may be either mobile or stationary)

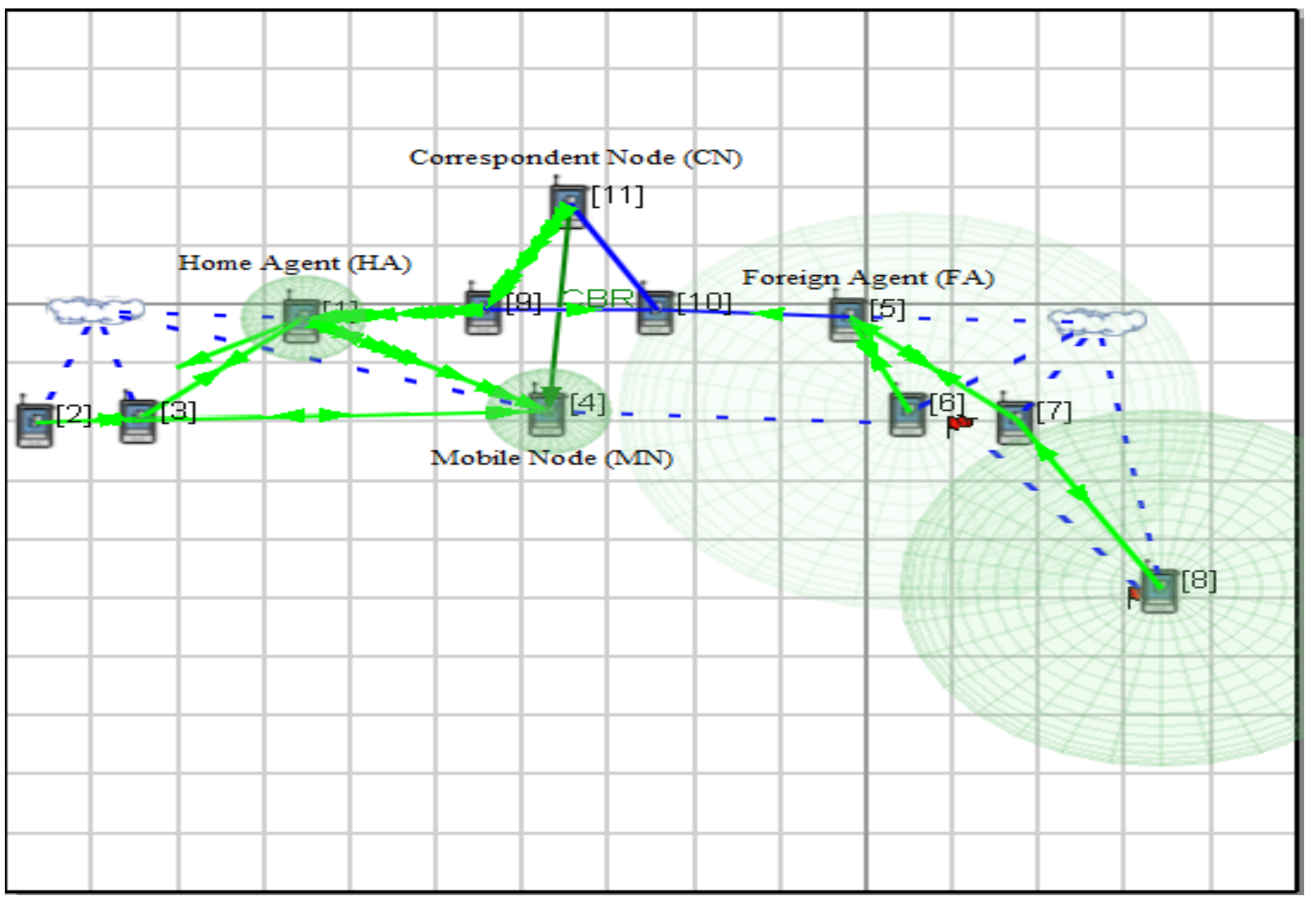

Fig. 1: Qualnet Simulated example of processes involved in Handover

Processes Involved during Handover are:-

- Agent Solicitation.

- Router Discovery.

- Address Configuration. 
- Movement Detection.

- Mobile IPv6 Registration.

\section{Contemporary Solutions}

To improve the Handover process various techniques are described in this section to improve Handover process and quality of services. The various techniques are as follows:-

\subsection{L3 (Network Layer) Driven Fast Handover Approach}

The Handover is the main concern in MIPv6.The bottleneck of MIPv6 is due to the fact that is that network layer use only the network layer information to detect whether the handover had taken place or not. To solve this problem several fast handover approaches came in to lime light which started using link layer information (L2) to speed the process of handover (Kazutaka et al, 2009).

There are two sub-processes related to the main handover approach:

- $\quad$ Link-Layer Handover (L2)

In this handover, the Mobile Node comes to know about change of Access Router as Mobile Node detects link layer address change to which it is linked with.

- Network layer Handover (L3)

L3 handover consists of two phases:

- Preparation Phase In this phase a CoA for mobile is generated as well as Duplicate Address Detection (DAD) protocol is executed.

- $\quad$ Signaling Phase

In this phase the CoA is registered with its Home Agent.

The evolution of L3- Driven handover was not at all abrupt. First of all Normal Handover Sequence was followed then after some time, Handover Sequence was developed Using L2 information. It was followed for some time and then L3- Driven handover was developed which was, indeed, more efficient than the other two previous approaches.

\subsubsection{Normal Handover Sequence}

First of all L2 handover will take place, whose functioning is not at all known to network layer. After a while the MN would receive a Router Advertisement and only then the L3 handover would start its sub phases: preparation phase and signaling phase will occur.

\subsubsection{Handover sequence Using L2 Information}

As soon as the L2 Handover is finished, the link layer notifies the network layer of the end of L2 handover. After getting this notification, the network layer sends the Router Solicitation message to the Access Router and, consequently, Access Router starts sending router advertisement message (P. Sangheon et al., 2006; R. Koodli et al., 2007).

\subsubsection{L3- Driven Fast Handover using L2 Information}

As shown in the Fig. 2 the link-layer senses the link quality and it notifies the network-layer. As soon as the link is down below the threshold the network- layer correspondingly executes the preparation phase immediately. 


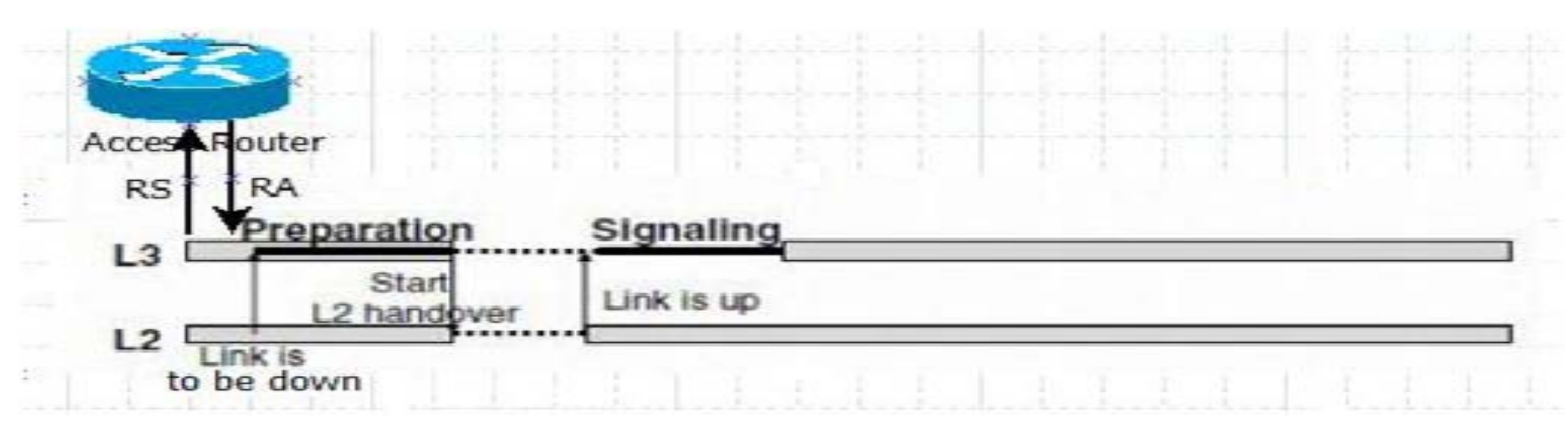

Fig. 2: L3-Driven Handover

As soon as the link layer finishes the L2 handover, it informs the network layer for executing its signaling phase.

It is important to know that the bottleneck of L3-Driven approach lies in being subservient to device dependent information. It is manifested through the radio wave strength by which to link layer can judge exact signal strength and notify the network layer that the link is down (N. Moore et al., 2006; Ming AI et al., 2008).

\subsection{Resource Efficient Care-of-Address Provisioning}

Duplicate Address Detection (DAD) protocol is followed so that each MN is using a unique address CoA i.e. no other node existed within the same domain subnet using the same global IP address (Care-of-Address) but it was found that DAD was a time consuming process (Longjiang Li et al, 2009), which might interrupt the seamless handover. in e.g. RFC 2462 DAD algorithm took more than 1000 millisecond to complete the DAD process which was not at all viable.

- After that a protocol called aDAD (advance DAD) was developed. The major advantage of aDAD is that it reserves unique New Care-of-Address in advance, as a result, it almost eliminated the latency needed for the Address Configuration and Confirmation .Another major advantage of aDAD is that it follows a concept called "Piggybacking" in accordance with which the New CoA request option is evolved through solicitation and New CoA Reply option through Router Advertisement is sent and received at one go. i.e. the MN sends a message called Router Solicitation to the Access Router and then the Access Router reverts the same message giving reply in the form of Router Advertisement. The major drawback of this protocol is that it uses excessively a lot of network resources such as bandwidth to generate new CoA and verify its uniqueness as aDAD generates New CoA and checks their uniqueness one by one (Deng et al, 2008).

- Third protocol is called Agent-based-DAD (XDAD).

It's the most efficient approach for providing New CoA, the advantage of this protocol is that it readily decreases the latency during handover. Another advantage is that it also reserves New CoA in an optimized and effective way. It generates New CoA and stores them in a cache.

XDAD uses two types of cache

- Active Proxy Cache.

- Active Garbage Cache.

Active Proxy Cache contains the newly generated CoA and the Active Proxy Garbage contains the CoA which MN relinquishes as soon as it leaves the subnet.

Actual Processes Involved In XDAD

- After receiving a solicitation message Access Router tries to reuse the CoA from the Active Proxy Garbage. If it doesn't get it from there, it uses store Active Proxy Cache address subsequently.

- Access routers then check the uniqueness of the generated CoA. If it is unique, it sends back the CoA within the same message as a Router Advertisement message through Piggybacking process. Otherwise Access Router drops the CoA and regenerates it.

- As soon as the CoA is assigned to a MN, CoA is deleted from the cache. 


\section{Proposed Solutions}

There are two modifications proposed in this sections these are described as follows:-

\subsection{In Domain Of L3-Driven Fast Handover Approach}

In the previously defined L3-Driven Fast Handover approach, there is need to frequently check whether link is down or not, and if the link is down that means that the present value of signal strength is below than threshold value.

- It is important to note here, that L3-Driven Handover requires some of the devices dependent information, so this dependence on the device for constant measuring and monitoring the signal strength is not at all required as it will frequently create problems such as wrong measurement of signal strength in a real dynamic environment may also cause havoc with entire mobility process.

- To remove this device dependent problem, the internal entities such as MN and Access Router should decide about the weak signal strength (Link). To know whether the link is down or not MN sends a packet to the Access Router and starts a timer, the router will revert the same packet back to the $\mathrm{MN}$, this process is done when $\mathrm{MN}$ is very much connected to the access router. The running timer will calculate the average time taken by the packet to traverse to and fro i.e. from MN to Access Router and from Access Router to the MN. Apropos of standard measurement of average time calculations will be repeated by sending the packets at least 5 times. Then the average of separate to and fro dispatching will be calculated and compared if these values remain same in all 5 remittances. Only then it will be set as standard time within the subnet.

- $\quad$ Now the MN will keep on moving and sending this packet. After some time, it will happen that packet will start taking more time to reach $\mathrm{MN}$, because when $\mathrm{MN}$ moves away beyond the reach of present Access Router, packets will not be able to reach the MN within set standard limit of the time.

- Now to enhance the efficiency of proposed modified version of MIPv6, it is also, envisaged that if the to and fro dispatch time is more than standard time and shows increasing trend of delay for 3 subsequent packets, then the device is so customized that it understands the feeble signal strength immediately and starts multicasting solicitation message to the adjacent router. for e.g. in Fig. 3 the to and fro dispatch of three consecutive dispatch are $2 \mathrm{~ms}, 7 \mathrm{~ms}, 10 \mathrm{~ms}$ as a result it will again watch the another set of 3 to and fro dispatch and if it shows the increasing trend in these values the handover will take place

- If the to and fro dispatch time of 3 subsequent packet for this router is less than the standard time, the MN immediately not perform the L2 handover., otherwise it will not happen, where as in Fig. 4 the to and fro dispatch of three consecutive dispatch are $2 \mathrm{~ms}, 10 \mathrm{~ms}, 3 \mathrm{~ms}$ so handover will not take place. This process finally eliminates the need of device-basedsignal-based-monitoring system and also overcome the delay on account of it.

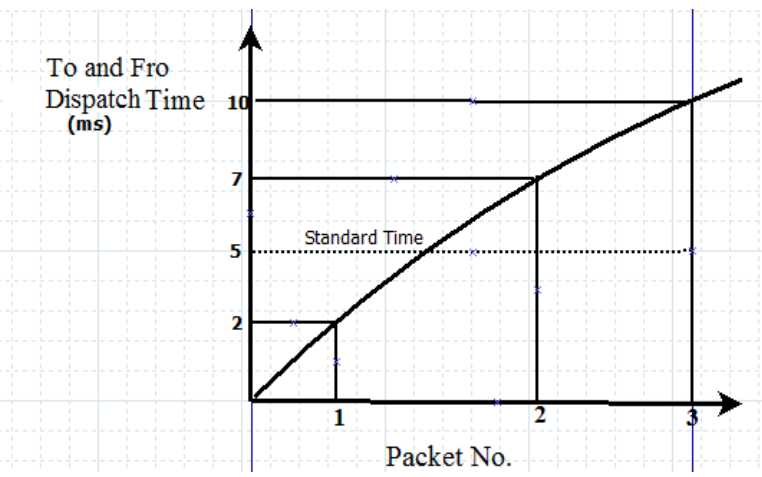

Fig. 3: Handover Required

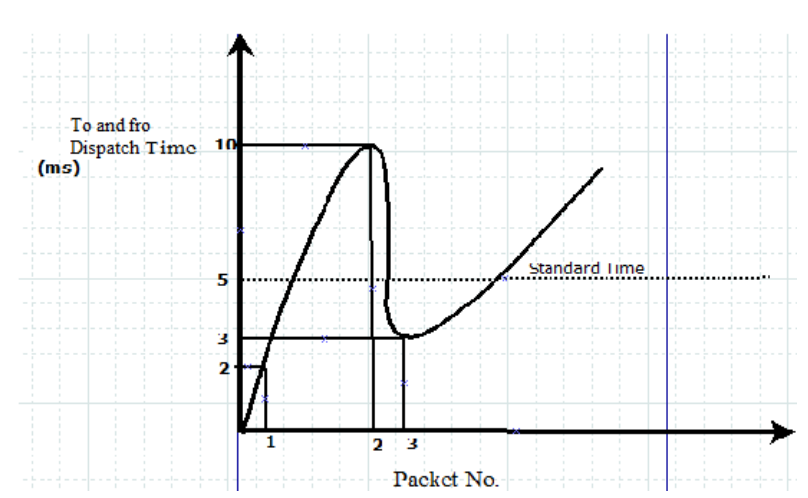

Fig. 4: No Handover Required

\subsection{In Domain of Resource Efficient CoA Provisioning}

In the previously defined Resource-Efficient CoA Provisioning two caches are used:

- Active Proxy cache.

- Active Proxy Garbage.

- These two caches are being managed by the Access Routers which will definitely increase to its overhead. To subside the overhead, it is recommended to reduce the no. of cache to one. Now, instead of using two caches, scope is reduced to one cache and names this cache as Working Cache. This Working Cache will store and allocate new unique CoA to the MN.

- The allocated CoA will be removed from the Working Cache and as soon as the MN leaves the subnet, again that CoA is reallocated to the Working Cache for reuse purpose. 
- The CoA is generated by the help of an algorithm and stored in the Working Cache. The algorithm will always generate a unique CoA that will remove the need of running DAD protocol and multicasting Neighbor Solicitation messages as every CoA is unique. If the need of running DAD protocol and multicasting Neighbor Solicitation is removed, it will definitely reduce the latency.

- Now the question arises that if the same algorithm is used by all the Access Routers to create unique CoA then what will differentiate the CoA if MN moves from one network to another and it will again get the same CoA in another Access Router. To resolve this problem use of the Link-Layer Address Prefix is suggested as it is very much unique for a subnet. The Prefix will be attached to the CoA and that's what will make it Unique within the 135subnet and outside the subnet.

\section{Conclusion}

To sum up, worth mentioning is the fact that Fast handover is one of the major challenge for the MIPv6 .To reduce this handover latency and utilize the present resources at their best two techniques (L3- Driven Fast Handover and Resource Efficient Care-ofAddress Provisioning) were discussed. But these techniques (L3- Driven Fast Handover and Resource Efficient Care-of-Address Provisioning) also are subjected to some limitations. The paper proposes that if these are addressed, to they could yield better results. This paper not only discusses the problem areas of the existing techniques but also proposes some concrete solutions to current problems to enhance their capabilities in terms of latency and better utilization of their resources and to enhance their efficiency for the future objective of fast and real dynamic world applications. Hopefully, if the proposed radical changes are incorporated in the existing MIPv6, it will not only bring about large scale viability in the system but also make it cost-effective, robust, and dynamic all the more.

\section{References}

Bi-Lynn Ong, Suhaidi Hassan,2007.Interworking of Protocols in IPv6 Mobility Management, IEEE International Conference on Telecommunications and Malaysia International Conference on Communications 2007.

Christian Vogt, 2009.A Comprehensive and Efficient Handoff Procedure for IPv6 Mobility Support,Proceedings of the 2006

International Symposium on a World of Wireless, Mobile and Multimedia Network (WoWMoM'o0),IEEE 2009.

Johnson D., Perkins C., and Arkko J., 2011.Mobility support in IPv6. RFC 6275.

Deng Ya-ping, Wu Ying-qiu,2008.Research on HMIPv6 Handover Latency of Improved DAD Policy. vol.2775, pp.679-691, Springer-Verlag

Kazutaka Gogo, Rie Shibui, Fumio Teraoka, 2009.An L3-Driven Fast Handover Mechanism in IPv6 Mobility,Proceedings of the International Symposium on Applications and the Internet Workshops(SAINTW 09)

Longjiang Li, Yuming Mao, Yonggang Li ,2009 .Resource-efficient Care-of Address Provisioning for Seamless IPv6 Mobility

Support, IEEE 802.21 Media Independent Handoff Working Group.

Ming AI, 2008. Design and Evaluation of A New Movement Detection and Address Configuration Method for supporting

Seamless Mobile IPv6 Handover, IEEE Proceedings of the International Symposium on a World of Wireless.

Moore N., 2006. Optimistic Duplicate Address Detection (DAD) for IPv6, IETF RFC 4429.

Calhoun P., Loughney J., Guttman E., Zorn G., and Arkko J., 2002.Diameter base protocol, draft-ietf-aaa-diameter-17.txt.

Sangheon P. and Yanghee C., 2006.Performance analysis of fast handover in Mobile IPv6 networks, Lecture Notes in Computer

Science (LNCS), vol.2775, pp.679-691, Springer-Verlag

Koodli R., 2007. Fast Handovers for Mobile IPv6, RFC 4068.

\section{Biographical notes:}

Mr.Purnendu Shekhar Pandey is a M.Tech Scholar at Gautam Buddha University. The author has done his B.Tech from Noida Institute of Engineering and Technology, Uttar Pradesh Technical University, Uttar Pradesh. The author has published and presented various papers in national and international Conferences/Journals.

K.S. Verma received his B. Tech. (Hons) in Electrical Engineering and M. Tech. in Electrical Engineering (Power Systems) both from KNIT Sultanpur (India) in1987 and 1998, respectively. He obtained his Ph.D. degree in Electrical Engineering (Power Systems) from Indian Institute of Technology, Roorkee (India) in 2003. Presently, Prof Verma is Director, KNIT Sultanpur (India). His research interests include FACTS Technology, distributed generation, power system optimization \& control, power quality and AI application in power system. 